\title{
Antidiarrheal action of Zataria multiflora hydroalcoholic and hexane extracts in mice
}

\author{
Hassan Sadraei ${ }^{{ }^{*}}$, Gholamreza Asghari ${ }^{2}$, Hossien Jamali ${ }^{1,2}$ \\ ${ }^{1}$ Department of Pharmacology \& Toxicology, School of Pharmacy and Pharmaceutical Sciences, Isfahan University of Medical Sciences, Isfahan, Iran \\ ${ }^{2}$ Department of Pharmacognosy, School of Pharmacy and Pharmaceutical Sciences, Isfahan University of Medical Sciences, Isfahan, Iran
}

\section{A R T I C L E I N F O}

Article Type:

Original Article

\section{Article History:}

Received: 6 June 2017

Accepted: 19 October 2017

\section{Keywords:}

Zataria multiflora

Diarrhea

Antispasmodic

Castor oil

$\mathrm{MgSO}_{4}$

\begin{abstract}
A B S T RA C T
Introduction: Zataria multiflora Boiss. is an indigenous herbal plant found in many parts of Iran. This herb is traditionally used as a remedy for treating gastrointestinal disorders including diarrhea. Despite the existence of few pharmacological evidences which support the antispasmodic action of $Z$. multiflora in vitro, there is no scientific report about therapeutic efficacy of $Z$. multiflora in animal models. The objective of this research was to investigate the antispasmodic activity of hydroalcoholic and hexane extracts of $Z$. multiflora on intestinal peristaltic movement as well as assessment of its antidiarrheal action in mice.

Methods: Dried leafy branches of Z. multiflora were coarsely powdered and subjected to extraction by ethanol or hexane in a percolator apparatus. Antispasmodic activity of $Z$. multiflora in vivo was assessed by investigating effect of the extracts on intestinal charcoal meal transit. The antidiarrheal activity of $Z$. multiflora extracts was evaluated by castor oil and magnesium sulfate-induced diarrhea. The inhibitory effects of the extracts were compared with the standard drug loperamide. Results: The antispasmodic activity of Z. multiflora $(20 \& 40 \mathrm{mg} / \mathrm{kg})$ hydroalcoholic and hexane extracts was confirmed by a reduction in the distance traveled by charcoal meal alongside the small intestine. Z. multiflora extracts ( $20 \& 40 \mathrm{mg} / \mathrm{kg}$ ) also significantly attenuated the castor oil and magnesium sulfate-induced diarrhea. Loperamide was more efficacious in reducing number of total stools in both models of diarrhea.

Conclusion: The obtained results have established a pharmacological evidence for the folkloric use of the Z. multiflora as an antidiarrhoeal and spasmodic agent.
\end{abstract}

Implication for health policy/practice/research/medical education:

Zataria multiflora hydroalcoholic and hexane extracts significantly inhibited small intestinal transit and reduced the severity of induced diarrhea and delayed the induction of diarrhea in animal models. These results are in consistent with traditional use of Z. multiflora for treatment of gastrointestinal disorders and show promising hope for preparation of a new drug.

Please cite this paper as: Sadraei H, Asghari G, Jamali H. Antidiarrheal action of Zataria multiflora hydroalcoholic and hexane extracts in mice. J Herbmed Pharmacol. 2018;7(1):22-28. doi: 10.15171/jhp.2018.05.

\section{Introduction}

Zataria multiflora Boiss. (Family Labiatae) is a medicinal plant which grows in Iran, Pakistan and Afghanistan (1). In Iran this plant is known as Avishan Shirazy and mainly used as food flavoring agent (1). This plant has several traditional medicinal uses including treatment of fever, labor pain, bone and joint pain, headache, migraine, common cold, bloating, nausea, airways obstruction and diarrhea (2). As Z. multiflora is a popular medicinal plant among people, its pharmacological properties have been reviewed in a number of scientific reports. There is substantial evidence for the efficacy of this medicinal plant for treatment of ailments such as pain, infection, inflammation, intestinal spasm, diabetes, and protecting liver function (3-8). Research studies revealed that both essential oil and hydroalcoholic extract of $Z$. multiflora contain pharmacologically active substances. Main constituents of the essential oil are thymol, $p$-cymene, carvacrol, linalool, $\gamma$-terpinene, trans-caryophyllene, $\alpha$-pinene, $\beta$-pinene, carvacrol methyl ether, borneol, cissabinene hydrate, 1,8-cineole, $\alpha$-terpinene, $a$-terpinolene, geraniol, eugenol, terpinene-4-ol, spathulenol, and 
camphor (9). Thymol is the most abundant compound among all constituents. It has been shown that essential oil of Z. multiflora subsides inflammatory signs of irritable bowel syndrome (1). The hydroalcoholic extract compositions are aliphatic phenols, alcohols, flavonoid, saponins and tanens. Also, apigenin, dihydroxy aromadendrene, a-tocopherolquinone, luteolin, and 6-hydroxyluteolin glycosides, as well as di-, tri-, and tetramethoxylated are compounds which have been identified in the $Z$. multiflora extracts (10).

Zataria multiflora has been traditionally used for the treatment of a number of gastrointestinal disorders including, stomachache, colitis and diarrhea (1). The anti-colitis action of the $Z$. multiflora has already been shown in animal models of colitis (11). Antispasmodic effect of $Z$. multiflora extract on smooth muscle spasm of rat ileum and uterus has been reported $(3,12)$. However, so far the inhibitory effect of $Z$. multiflora extract on intestinal spasm in vivo has not been reported. Therefore, in this research we investigated the gastrointestinal effect of $Z$. multiflora hexane and hydroalcoholic extracts on intestinal movement as well as their antidiarrheal potentials in animal models.

\section{Materials and Methods}

Plant material and extraction

Aerial parts of $Z$. multiflora were collected from Ardestan region in spring 2016 (Isfahan, Iran). The plant was dried in shade at room temperature. The plant was identified as Z. multiflora and a specimen was deposited at botany herbarium of School of Pharmacy and Pharmaceutical Sciences (reference No. 1483). The stiff branches were separated and the rest was coarsely powdered by an electronic grinder (Moulinex, France) and then subjected to extraction in a percolator.

For the preparation of the hydroalcoholic extract, $200 \mathrm{~g}$ of dried and powdered Z. multiflora was percolated with $70 \%$ ethanol. To obtain the non-polar fractions, a powdered sample of $Z$. multiflora was extracted with n-hexane. The plant powder was percolated for 24 hours according to pharmacopoeia reference $(13,14)$. The solvents were eliminated from the extracts by evaporation under vacuum in a rotary evaporator (Buchi Rotavapor RE) at $50^{\circ} \mathrm{C}$. The crude extracts were stored in a refrigerator. A sample of the hydroalcoholic extract was totally dried on a heater for determining the percentage of remaining water.

Animals and solutions

Albino male mice (22-28 g) were purchased from School of Pharmacy animal house and handled according to university guidelines for animal care and handling (15). All animals were fasted overnight before starting the experiment with free excess to water ad libitum.

The extracts were prepared as described above. Loperamide and extracts were made up in $70 \%$ ethanol as $20 \mathrm{mg} / \mathrm{mL}$ stock solution. Further dilutions were made in distilled water as appropriate. Sulfate magnesium solution was prepared as $10 \%$ stock solution. For the preparation of charcoal meal tragacanth suspension (5\%) was mixed with charcoal suspension (3\%) solution. Unless stated, all chemicals were from Merck (Germany).

\section{Gastrointestinal transit test}

Animals were fasted for overnight and divided into seven groups. Mice in groups I and II received oral administration of loperamide solution $(2 \mathrm{mg} / \mathrm{kg})$ or vehicle $(0.35 \%$ ethanol). Groups III and IV received the hydroalcoholic extract $(20 \mathrm{mg} / \mathrm{kg}$ and $40 \mathrm{mg} / \mathrm{kg}$ p.o.). Groups $\mathrm{V}$ and VI received the hexane extract (20 and $40 \mathrm{mg} / \mathrm{kg}$ p.o.). The animals in control group (VII) were treated with equivolume amount of the vehicle ( $7 \%$ ethanol). $0.5 \mathrm{~mL}$ of charcoal meal was administered orally using steel feeding tube after 30 minutes and the animals were left in the cage. Forty-five minutes after oral administration of charcoal meal, each animal was anesthetized with diethylether and sacrificed. The entire intestine was carefully dissected out and lined up on the table. The total length of the small intestine and the distance that charcoal meal had traveled was measured. The volume of administrated solution was adjusted as such that all animals were received $0.5 \mathrm{~mL}$ of drug or vehicle.

\section{Castor oil-induced diarrhea}

Animals were fasted overnight and divided into seven groups as above. On the day of experiment $0.5 \mathrm{~mL}$ of hydroalcoholic extract, hexane extract or loperamide were given orally to the animals $(\mathrm{n}=10)$. The animals in control group were treated with equivolume amount of vehicle as above. Half an hour later, diarrhea was induced by oral administration of castor oil $(0.5 \mathrm{~mL})$. Each animal was placed on a tissue paper under a glass funnel and observed for any wet defecation for a period of 3 hours.

\section{Magnesium sulfate-induced diarrhea}

A similar protocol as for castor oil-induced diarrhea was followed. Diarrhea was induced by oral administration of $\mathrm{MgSo}_{4}(2 \mathrm{~g} / \mathrm{kg})$. Half an hour later, the effect of $Z$. multiflora hydroalcoholic and hexane extracts were examined on diarrhea induced by $\mathrm{MgSO}_{4}$ and compared with the standard drug loperamide. The animals in control group were treated with vehicle.

\section{Measurement and statistics analysis}

Gastrointestinal transit was expressed as the percentage of distance that charcoal moved relative to the whole length of small intestine. Diarrhea was scored as the number of watery defecation at appropriate time. The absorbent paper was changed regularly and number of wet defecations was recorded. Number of diarrheic feces excreted at 30min intervals as well as total wet fecal outputs was calculated. Delay in the induction of diarrhea was the time when first sing of diarrhea was observed following administration of 
laxatives. Mean and standard error of mean (SEM) were calculated for each group of results and compared with the control groups using unpaired Student's $t$ test. SigmaPlot software (version 11) was used for statistical analysis and plotting the graphs.

\section{Results}

Plant extraction

Concentrated hydroalcoholic extract had a browngreenish color. The water content was calculated to be $20 \%$. The yield of hydroalcoholic extract was $12 \%$ (W/W). The hexane extract has brownish color and its yield was $1 \%(\mathrm{~W} / \mathrm{W})$.

\section{Gastrointestinal transit test}

In the control group treated with vehicle, the charcoal meal covered up to about three quarters of the entire small intestine (Figure 1). Loperamide $(2 \mathrm{mg} / \mathrm{kg}$ ) significantly reduced the charcoal meal transit by $67 \%$ (Figure 1 ). The hydroalcoholic extract with oral doses of $20 \mathrm{mg} / \mathrm{kg}$ and $40 \mathrm{mg} / \mathrm{kg}$ reduced gastrointestinal transit by $14 \%$ and $40 \%$ respectively (Figure 1 ). Similarly the hexane extract attenuated the charcoal meal transit by $33 \%$ and $49 \%$ with oral doses of $20 \mathrm{mg} / \mathrm{kg}$ and $40 \mathrm{mg} / \mathrm{kg}$, respectively (Figure 1).

\section{Castor oil-induced diarrhea}

Mice in the control group produced copious diarrhea following oral administration of castor oil. First sign of diarrhea was observed within first half an hour after castor oil administration and diarrhea reached its peaks about 75 minutes and thereafter gradually subsided down (Figure 2a). On the other hand, animals pretreated with hydroalcoholic extract of $Z$. multiflora and hexane extract of $Z$. multiflora showed a significant delay in

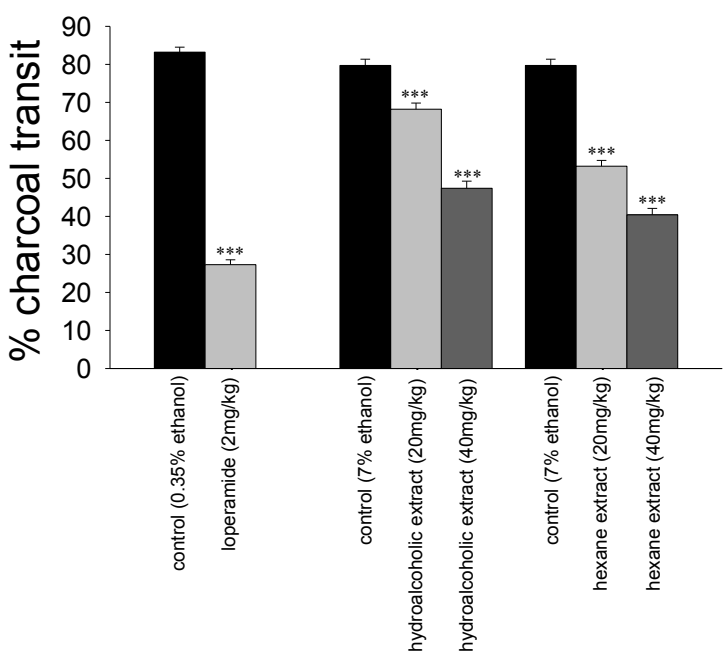

Figure 1. Effect of loperamide, Zataria multiflora hydroalcoholic and hexane extracts on small intestinal transit $(0.5 \mathrm{~mL}$ of charcoal meal). Data are presented as mean $\pm \operatorname{SEM}(n=10)$. Stars show degree of statistically significant difference in comparison with its appropriate control group $\left({ }^{* * *} P<0.001\right.$, Student's $t$ test). onset of diarrhea (Table 1), decrease in the frequency of wet defecation (Figure 3) and the total wet stool recorded over 3 hours time (Figure 4). Few mice showed no sign of diarrhea after treatment with loperamide or extracts. Loperamide reduced the incidence of total wet defecation by $80 \%$ while hydroalcoholic extract of $Z$. multiflora with doses of $20 \mathrm{mg} / \mathrm{kg}$ and $40 \mathrm{mg} / \mathrm{kg}$ reduced the incidence of total wet discharge by $49 \%$ and $63 \%$, respectively in comparison to their corresponding control groups. Similarly hexane extract $(20 \& 40 \mathrm{mg} / \mathrm{Kg}$ ) reduced the total number of wet defecation by $56 \%$ and $73 \%$, respectively over the course of the study (Figure 4).

Magnesium sulfate-induced diarrhea

In the control group treated with the vehicle, oral administration of $\mathrm{MgSO}_{4}$ induced watery stool discharge in all the animals. Time of induction and the peak of diarrhea followed a similar pattern as it was seen with the castor oil (Figure 5). However, the severity of observed watery defecation was less copious than those seem with the castor oil. Loperamide significantly delayed the onset of diarrhea (Table 1) and two mice had no sign of diarrhea

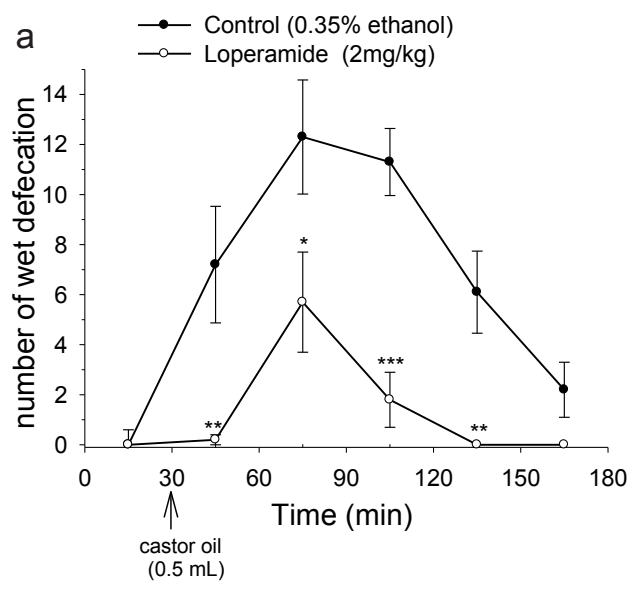

b

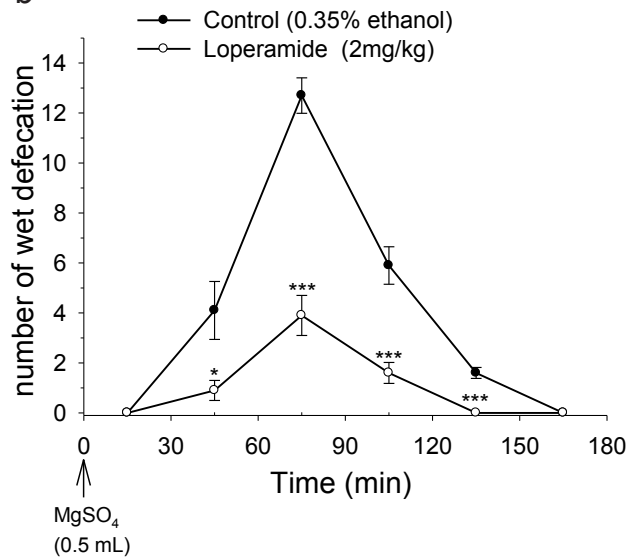

Figure 2. Antidiarrheal activity of loperamide with castor oil (0.5 $\mathrm{mL}$ p.o) and $\mathrm{MgSO}_{4}(0.5 \mathrm{~mL}, 10 \%$ solution) induced diarrhea. Incident of diarrhea were assessed as number of wet defecation at 30 min intervals. Data are shown as mean \pm SEM, $n=10$ for each group. Key: ${ }^{* *} P<0.01,{ }^{* * *} P<0.001$ in comparison with corresponding vehicle treated control group (Student's $t$ test). 
Table 1. Effect of Zataria multiflora extracts on induction of diarrhea following oral administration of castor oil and magnesium sulfate in mice

\begin{tabular}{llcc}
\hline \multirow{2}{*}{ Treatment } & Dose & \multicolumn{2}{c}{$\begin{array}{c}\text { Delay in induction of } \\
\text { diarrhea (min) }\end{array}$} \\
\cline { 3 - 4 } & & Castor oil & MgSO $_{4}$ \\
\hline Control group & $7 \%$ ethanol & $50 \pm 4.6$ & $54 \pm 3.2$ \\
Hydroalcoholic extract & $20 \mathrm{mg} / \mathrm{kg}$ & $70 \pm 9.5$ & $62 \pm 3.6$ \\
Hydroalcoholic extract & $40 \mathrm{mg} / \mathrm{kg}$ & $79 \pm 11^{*}$ & $61 \pm 3.4$ \\
Hexane extract & $20 \mathrm{mg} / \mathrm{kg}$ & $63 \pm 3.8^{*}$ & $56 \pm 3$ \\
Hexane extract & $40 \mathrm{mg} / \mathrm{kg}$ & $70 \pm 5^{* *}$ & $674.5^{*}$ \\
Control group & $0.35 \%$ ethanol & $54 \pm 6.5$ & $54 \pm 3.2$ \\
Loperamide & $2 \mathrm{mg} / \mathrm{kg}$ & $75 \pm 4.8^{*}$ & $66 \pm 5.2^{*}$ \\
\hline
\end{tabular}

All data are expressed as mean \pm SEM. for each group $(n=10) . * P<0.05$, $* * P<0.01$ in comparison with corresponding control group (Student's $t$ test).
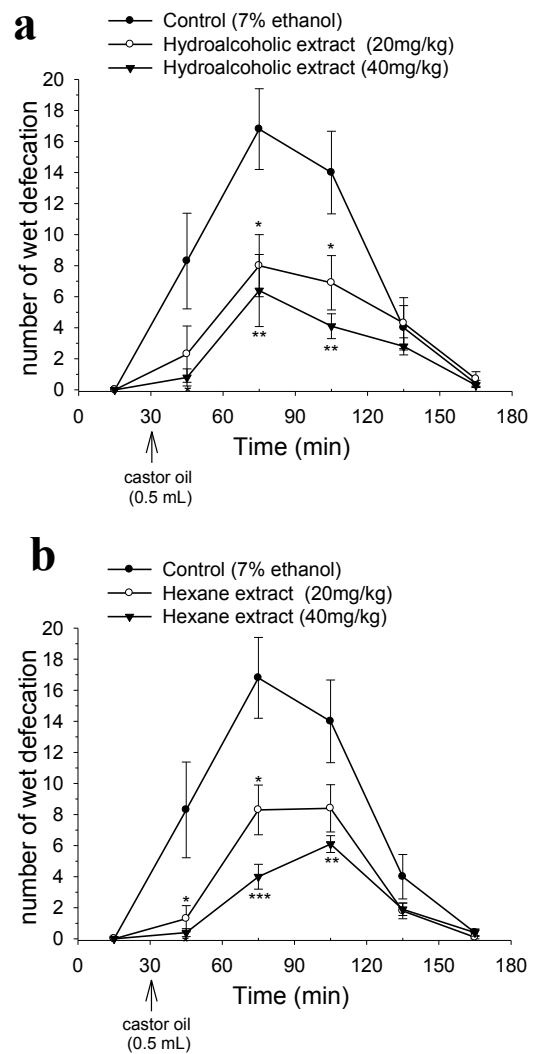

Figure 3. Antidiarrheal activity of Zataria multiflora hydroalcoholic (a) and hexane (b) extracts on induced diarrhea by castor oil ( 0.5 $\mathrm{mL}$ p.o). The incidence of diarrhea was assessed as number of wet defecation at $30 \mathrm{~min}$ intervals. Data are shown as mean \pm SEM $(\mathrm{n}=10)$. Key: ${ }^{*} \mathrm{P}<0.05,{ }^{* *} P<0.01,{ }^{* * *} P<0.001$ in comparison with vehicle treated control group (Student's $t$ test).

at all. Loperamide significantly reduced number of wet defecation by $73 \%$ in comparison to the vehicle treated control group (Figure 6). Hydroalcoholic and hexane extracts of $Z$. multiflora (20 \& $40 \mathrm{mg} / \mathrm{kg}$ ) significantly reduced the frequency of wet defecation (Figure 5). The incidence of total wet discharge was reduced by $33 \%$ and $48 \%$ in the test group treated with a dose of $20 \mathrm{mg} /$ $\mathrm{kg}$ of hydroalcoholic or hexane extracts, respectively. The

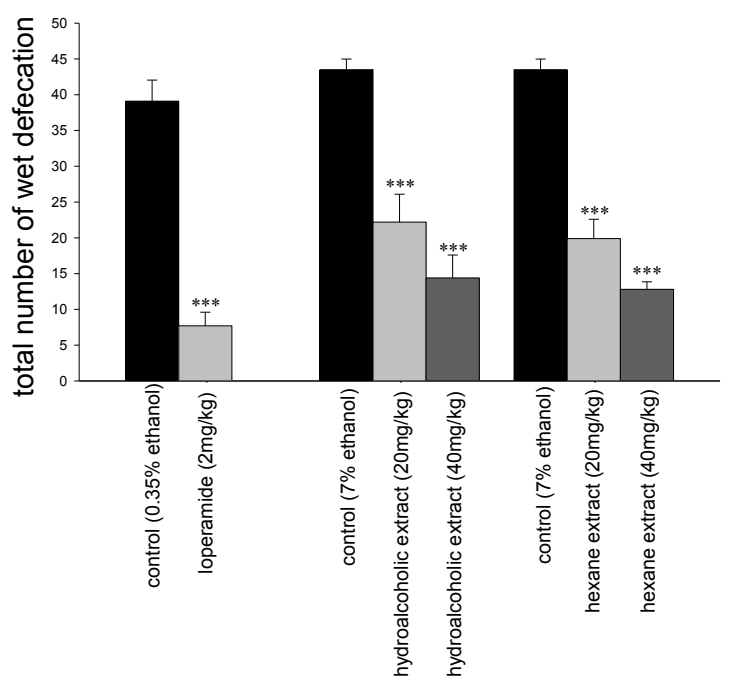

Figure 4. Antidiarrheal activity of loperamide, Zataria multiflora hydroalcoholic and hexane extracts on induced diarrhea by castor oil $(0.5 \mathrm{~mL}$ p.o). Each bar shows the total number of wet defecation following oral administration of castor oil. Data are presented as mean \pm SEM $(n=10)$. Key: ${ }^{* * *} P<0.001$ in comparison with the corresponding vehicle treated control group (Student's $t$ test).
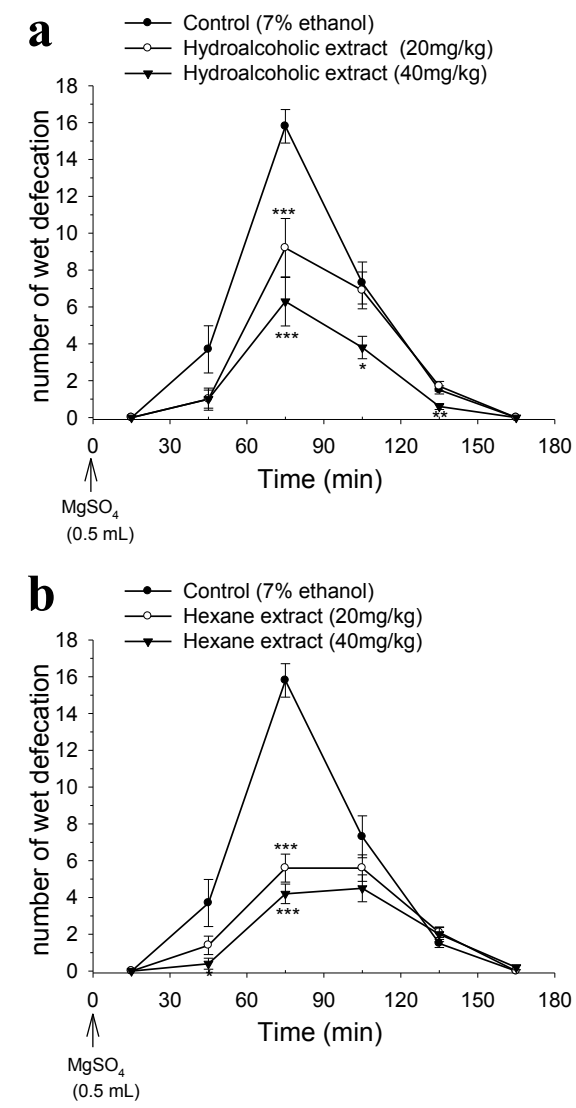

Figure 5. Antidiarrheal activity of Zataria multiflora hydroalcoholic (a) and hexane (b) extracts on induced diarrhea by $\mathrm{MgSO}_{4}(0.5$ $\mathrm{mL}, 10 \%$ solution). Incidences of diarrhea were assessed as number of wet defecation at 30 min intervals. Data are as mean \pm SEM $(n=10)$. Key: ${ }^{*} P<0.05,{ }^{* \star} P<0.01,{ }^{* * *} P<0.001$ in comparison with vehicle treated control group (Student's t-test). 


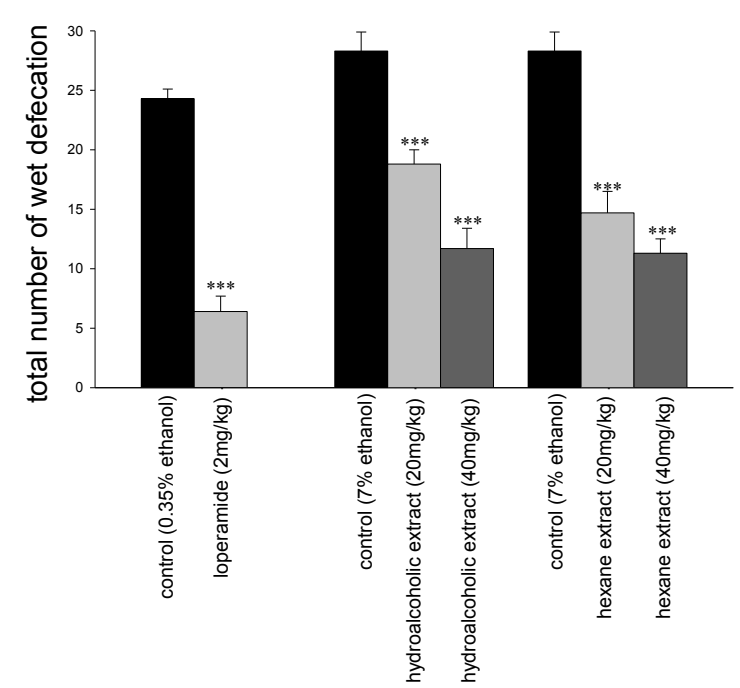

Figure 6. Antidiarrheal activity of loperamide, Zataria multiflora hydroalcoholic and hexane extracts on induced diarrhea by $\mathrm{MgSO}_{4}(0.5 \mathrm{~mL}, 10 \%$ solution$)$. Each bar shows the total number of wet defecation following oral administration of $\mathrm{MgSO}_{4}$. Data are presented as mean \pm SEM $(n=10)$. ${ }^{* * *} P<0.001$ in comparison with the corresponding vehicle treated control group (Student's $t$ test).

severity of the diarrhea was further reduced by increasing the oral dose of the extracts to $40 \mathrm{mg} / \mathrm{kg}$ (Figure 5) and the total number of wet defecation over the course of the study was reduced by $58 \%$ and $60 \%$, respectively (Figure 4 ). Loperamide $(2 \mathrm{mg} / \mathrm{kg}), Z$. multiflora hydroalcoholic and hexane extracts $(20 \& 40 \mathrm{mg} / \mathrm{kg})$ significantly inhibited small intestinal transit and reduced the severity of induced diarrhea and delayed the induction time of diarrhea.

\section{Discussion}

Diarrhea has long been recognized as a common disease of tropical areas and has been responsible for morbidity and mortality of children and infants, especially in developing countries (16). The World Health Organization on its Diarrhoeal Diseases Control Program has emphasized on the use of traditional medicine in order to reduce diarrhea morbidity and mortality (16). Diarrhea is mainly associated with impairment of fluid and electrolyte absorption or intense increase in intestinal motility (17). Herbal medicines have long been used as a remedy for the treatment of gastrointestinal disorders including diarrhea (2). Z. multiflora is a medicinal plant which has been used for gastrointestinal discomfort in Asia (2). The main goal of this research was to investigate the antidiarrheal effect of $Z$. multiflora extract in animal models. In vivo antidiarrhoeal activity of extracts was assessed using castor oil and $\mathrm{MgSO}_{4}$-induced diarrhea (18). Castor oil is known as a stimulant laxative. It works by increasing the movement of the intestines. Castor oil is non-irritant, but in small intestine it is hydrolyzed to ricinoleic acid which has a strong irritant effect on the gut mucosa (19). Ricinoleic acid induces diarrhea by releasing nitric oxide (NO), stimulating prostaglandin synthesis and increasing peristalsis (20). On the other hand, $\mathrm{MgSO}_{4}$ is an osmotic laxative (18). $\mathrm{MgSO}_{4}$ induces diarrhea by promoting cholecystokinin release from the duodenal mucosa preventing the reabsorption of sodium chloride and water from the lumen (18). Loperamide had profound antidiarrheal activities on both castor oil and $\mathrm{MgSO}_{4}$-induced diarrhea. Loperamide is opioid receptor agonist and acts on $\mu$-receptor on both intestinal smooth muscle and on the nerve terminal innervated gut. This inhibits intestinal peristaltic activity and reduces water and electrolyte secretion (21). Reduction in intestinal motility is associated with inactivating $\mathrm{Ca}^{2+}$ ion influx through voltage gated $\mathrm{Ca}^{2+}$ channels (22). Reduction in neurotransmitters release, especially acetylcholine, is due to activation of presynaptic opioid receptors which act as autoreceptor on cholinergic nerve terminals $(21,22)$. As intestinal peristaltic movement is mostly controlled by parasympathetic activities, slowing of intestinal movement by loperamide could also be due to reduction in acetylcholine release. Attenuation of parasympathetic activities allows more time for water absorption in gastrointestinal tract. Suppression of $\mathrm{MgSo}_{4}$-induced diarrhea by loperamide could also be explained by inhibition of acetylcholine release and reduction of electrolyte secretions.

Pretreatment of mice with both hydroalcoholic and hexane extracts of $Z$. multiflora provided significant protection against castor oil induced diarrhea. Comparison of antidiarrhoeal action of hydroalcoholic and hexane extract shows that they have a relatively similar effect and this may indicate that the antidiarrhoeal compounds exist in both types of extracts. Hexane extract mainly contain the non-polar constituents while both polar and nonpolar constituents exist in hydroalcoholic extract. This may indicate that the active components mainly have non-polar properties. Apigenin has been identified as one the active compounds of $Z$. multiflora extract (10). In another research we have reported the antidiarrheal action of apigenin (23). However, it is likely that other active ingredients exist which need to be identified.

In the case of $\mathrm{MgSO}_{4}$, the extracts were given after induction of diarrhea. Both hydroalcoholic and hexane extracts of $Z$. multiflora reduce severity and number of diarrhea incidence. Within 45 minutes following oral administration of $Z$. multiflora extract, the antidiarrheal action of the extract was visible. This shows that the active ingredient has relatively fast action. When the antidiarrheal doses of the extracts are compared with that of loperamide, it looks that loperamide is more potent the extract. However, the extract is composed of unknown numbers of active and inactive constituents. With disregard of inactive substance it is likely that antidiarrheal activity of $Z$. multiflora extract will not be less than loperamide. Therefore, isolation and identification of all active ingredients of $Z$. multiflora extract are recommended. 
Measurement of charcoal meal transit is used as an indication of intestinal peristaltic movement. Hydroalcoholic extract of $Z$. multiflora has potent inhibitory effect on isolated ileum contraction induced by acetylcholine, high potassium concentration and neuronal stimulation (3). Therefore, reduction in charcoal meal transit by hydroalcoholic extract of $Z$. multiflora was expected. Reduction in intestinal motility could also explain the antidiarrheal action of $Z$. multiflora extracts. As the hexane extract shows similar anti-motility activity, it is possible that similar components which exist in both types of extract are responsible for reduction in intestinal motility. Apigenin which is a known constituent of Z. multiflora also reduced intestinal motility under similar condition (23). Although apigenin could have a substantial participation in reduction of intestinal movement, however, the role of other substances such those exist in the essential oil should not be ignored. For instance it has been shown that essential oil components such as $\alpha$-pinene, $\beta$-pinene, $\alpha$-terpineol, geraniol have antispasmodic activities on isolated ileum (24-26). These components are also found in the essential oil of $Z$. multiflora.

\section{Conclusion}

In this research pharmacological evidence was presented for the effectiveness of $Z$. multiflora extracts as antispasmodic and antidiarrheal remedies. As the extract is a combination of active and inactive substances, separation and identification of the active ingredients for further investigations and development of lead compounds are recommended.

\section{Acknowledgements}

We would like to thanks the research department of Isfahan University of Medical Sciences, Isfahan, Iran for providing a student's grant (NO: 395854) for this research project.

\section{Authors' contributions}

GA was responsible for preparation of extracts while HS supervised the pharmacological studies. HJ was responsible for performing the experimental work.

\section{Conflict of interests}

The authors declare no conflict of interest.

\section{Ethical considerations}

Ethical issues have been observed by the authors.

\section{Funding/Support}

This research was financially supported by Research department of Isfahan University of Medical Sciences

\section{References}

1. Sajed H, Sahebkar A, Iranshahi M. Zataria multiflora
Boiss. (Shirazi thyme)--an ancient condiment with modern pharmaceutical uses. J Ethnopharmacol. 2013;145(3):68698. doi: 10.1016/j.jep.2012.12.018.

2. Naghibi F, Mosaddegh M, Mohammadi Motamed M, Ghorbani A. Labiatae Family in folk Medicine in Iran: from Ethnobotany to Pharmacology. Iran J Pharm Res. 2010;4(2):63-79.

3. Naseri MK. Effect of Zataria multiflora Boiss. leaf hydroalcoholic extract on rat ileum. J Kermanshah Univ Med Sci. 2003;7(3):1-4.

4. Hosseinzadeh H, Ramezani M, Salmani G. Antinociceptive, anti-inflammatory and acute toxicity effects of Zataria multiflora Boiss extracts in mice and rats. J Ethnopharmacol. 2000;73(3):379-85.

5. Shafiee A, Javidnia K, Tabatabai M. Volatile constituents and antimicrobial activity of Zataria multiflora, population Iran. Iran J Chem Eng. 1999;18(1):1-5.

6. Mohammadi A, Gholamhoseinian A, Fallah H. Zataria multiflora increases insulin sensitivity and PPARgamma gene expression in high fructose fed insulin resistant rats. Iran J Basic Med Sci. 2014;17(4):263-70.

7. Owlia P, Pirveicy H, Saderi H, Rezvani M, Mansouri S. Evaluation of the antimicrobial effects of extract of Zataria multiflora against oral Streptococci. Iran J Pharm Res. 2010;3(2):74-5.

8. Shokrzadeh M, Chabra A, Ahmadi A, Naghshvar F, Habibi E, Salehi F, et al. Hepatoprotective effects of Zataria multiflora ethanolic extract on liver toxicity induced by cyclophosphamide in mice. Drug Res (Stuttg). 2015;65(4):169-75. doi: 10.1055/s-0034-1370932.

9. Sadraei H, Ghannadi A, Takei-bavani M. Effects of Zataria multiflora and Carum carvi essential oils and hydroalcoholic extracts of Passiflora incarnata, Berberis integerrima and Crocus sativus on rat isolated uterus contractions. Int J Aromather. 2003;13(2):121-7. doi: 10.1016/S09624562(03)00092-4.

10. Ali MS, Saleem M, Ali Z, Ahmad VU. Chemistry of Zataria multiflora (Lamiaceae). Phytochemistry. 2000;55(8):933-6.

11. Nakhai LA, Mohammadirad A, Yasa N, Minaie B, Nikfar S, Ghazanfari G, et al. Benefits of Zataria multiflora Boiss in Experimental Model of Mouse Inflammatory Bowel Disease. Evid Based Complement Alternat Med. 2007;4(1):43-50. doi: 10.1093/ecam/nel051.

12. Gharib Naseri M, Mazlomi H, Goshaiesh M, Vakilzadeh G, Heidari A. Antispasmodic Effect of Zataria multiflora Boiss. Leaf Extract on the Rat Uterus. Iran J Pharm Res. 2010;5(2):131-6.

13. Ghasemi Dehkordi NA, Sajadi SE, et al. Iranian Herbal Pharmacopoeia. Ministry of Health Pub. Tehran. 2002;1:105.

14. Samuelsson G. Drugs of natural origin. Stockholm: Swedish Pharmaceutical Press: Sweden; 1999:48-9.

15. Committee for the update of the guide for the care and use of laboratory animals National Research Council. Guide for the Care and Use of Laboratory Animals. Washington DC: The National Academies Press; 2010:11-37.

16. Snyder JD, Merson MH. The magnitude of the global problem of acute diarrhoeal disease: a review of active surveillance data. Bull World Health Organ. 1982;60(4):605-13.

17. Rang HP, Dale MM, Ritter JM, Flower RJ. Rang and Dale's 
Pharmacology. 6th ed. London: Churchill Livingstone; 2007:385-96.

18. Izzo AA, Gaginella TS, Capasso F. The osmotic and intrinsic mechanisms of the pharmacological laxative action of oral high doses of magnesium sulphate. Importance of the release of digestive polypeptides and nitric oxide. Magnes Res. 1996;9(2):133-8.

19. Pasricha PJ. Treatment of disorders of bowel motility and water flux; antiemetics; agents used in biliary and pancreatic disease. In: Brunton LL, Lazo JS, Parker KL, eds. Goodman and Gilman's The Pharmacological Basis of Therapeutics. 11th ed. New York: McGraw-Hill; 2006:983-1008.

20. Kaur M, Singh A, Kumar B. Comparative antidiarrheal and antiulcer effect of the aqueous and ethanolic stem bark extracts of Tinospora cordifolia in rats. J Adv Pharm Technol Res. 2014;5(3):122-8. doi: 10.4103/2231-4040.137417.

21. Kromer W. Endogenous and exogenous opioids in the control of gastrointestinal motility and secretion. Pharmacol Rev. 1988;40(2):121-62.
22. Reynolds IJ, Gould RJ, Snyder SH. Loperamide: blockade of calcium channels as a mechanism for antidiarrheal effects. J Pharmacol Exp Ther. 1984;231(3):628-32.

23. Sadraei H, Asghari G, Shahverdi F. Antidiarrhoeal assessment of hydroalcoholic and hexane extracts of Dracocephalum kotschyi Boiss. and apigenin in mice. Res Pharm Sci. 2016;11(3):200-9.

24. Sadraei H, Asghari GR, Hajhashemi V, Kolagar A, Ebrahimi M. Spasmolytic activity of essential oil and various extracts of Ferula gummosa Boiss. on ileum contractions. Phytomedicine. 2001;8(5):370-6. doi: 10.1078/0944-711300052.

25. Sadraei H, Asghari G, Kasiri F. Comparison of antispasmodic effects of Dracocephalum kotschyi essential oil, limonene and alpha-terpineol. Res Pharm Sci. 2015;10(2):109-16.

26. Sadraei H, Asghari G, Emami S. Inhibitory effect of Rosa damascena Mill flower essential oil, geraniol and citronellol on rat ileum contraction. Res Pharm Sci. 2013;8(1):17-23. 